\title{
Editorial
}

\section{A New Phase, A New Look, A Long Life}

The first issue of a third volume of the journal is here at last. As many of you will be aware, much has happened since I wrote the last editorial that so naively celebrated the anniversary of its launch. The journal had already begun to rise up the Higher Education Academy (HEA) journal rankings. However, with very little warning, in July 2014 we heard that the HEA was discontinuing its journal portfolio; probably all part of the highs and lows of being a journal editor but for this to happen so soon was very disappointing.

However, never people to be beaten easily, Jill Thistlethwaite, Vinette Cross and I discussed options with the Chair of the National Association of Educators in Practice, Dr Jane Morris, and the Executive Committee. We agreed that we had invested too much energy in getting the journal established to close it down without a fight. Our primary aim was to maintain a journal that would be widely available free of charge to authors and readers, and to continue to provide opportunity to publish research that does not necessary fit comfortably with the aims, scope and readership of either general educational or profession specific journals. Several options were explored but all had drawbacks.

This was when we hit on the idea of using the Open Journal System. The learning curve has been vertical and we would not have got this far without the help of a great team of colleagues in the IT department, the Centre for Academic Writing and the Lanchester Library at Coventry University. We have also been supported by Dr Virginia King, a computer scientist, researcher and academic developer to whom we are indebted for her attention to our queries and for getting to grips with what is a complex submission, review and publication system.

We wish to thank the authors of the first volume of the new-look journal for their support in taking the decision not to withdraw papers already in the system, and for waiting patiently to see their work in print. Likewise, the efforts of reviewers who have begun to become familiar with the new system are much appreciated. We are delighted with the new issue and hope that you will find lots to interest you. The articles cross several different professions and reflect a wide range of issues, not least those that arise from interprofessional initiatives across continents. This issue contains articles from Australia, Britain, Ireland and the United States of America. Whilst planning forward, we are also acutely aware of the need to secure back copies of the journal and we are working with the HEA to negotiate to import previous issues to the Open Journal System, that incidentally, are still being accessed and downloaded actively.

The journal focus remains unchanged; it provides a forum for research, reflective and masterclass articles on any aspect of learning related to practice. We aim to publish two editions a year with an option to increase to three, if and when this is necessary. Readers can help by spreading the word about the journal, promoting the web address

http://tinyurl.com/pblhsc through email signatures and through social media. Future success depends on you, the practitioners, researchers and readers.

An apt metaphor springs to mind when I reflect on the journal's development so far: the mythical phoenix, which dies in a show of flames and combustion to be regenerated from the 
ashes, is adopted by Coventry University as its symbol. However, befitting the journal's developing profile and our aspiration to reach colleagues globally, its analogues can be found in a wide range of cultures including Arabic, Chinese, Greek, Hindu, Japanese, Roman, Russian and Turkish mythology giving its message wide appeal.

Typically, the phoenix lives a colourful, long and vibrant life! What more could we hope for?

Lynn Clouder

Coventry University, UK 